\title{
miR-139 modulates MCPIP1/IL-6 expression and induces apoptosis in human OA chondrocytes
}

\author{
Mohammad Shahidul Makki and Tariq M Haqqi
}

IL-6 is an inflammatory cytokine and its overexpression plays an important role in osteoarthritis (OA) pathogenesis. Expression of IL-6 is regulated post-transcriptionally by MCPIP1. The $3^{\prime}$ untranslated region (UTR) of MCPIP1 mRNA harbors a miR-139 'seed sequence', therefore we examined the post-transcriptional regulation of MCPIP1 by miR-139 and its impact on IL- 6 expression in OA chondrocytes. Expression of miR-139 was found to be high in the damaged portion of the OA cartilage compared with unaffected cartilage from the same patient and was also induced by IL-1ß in OA chondrocytes. Inhibition of miR-139 decreased the expression of IL- 6 mRNA by $38 \%$ and of secreted IL- 6 protein by $40 \%$. However, overexpression of miR-139 increased the expression of IL- 6 mRNA by $36 \%$ and of secreted IL- 6 protein by $56 \%$. These data correlated with altered expression profile of MCPIP1 in transfected chondrocytes. Studies with a luciferase reporter construct confirmed the interactions of miR-139 with the 'seed sequence' located in the 3' UTR of MCPIP mRNA. Furthermore, miR-139 overexpression increased the catabolic gene expression but expression of anabolic markers remained unchanged. Overexpression of miR-139 also induced apoptosis in OA chondrocytes. Importantly, we also discovered that IL- 6 is a potent inducer of miR-139 expression in OA chondrocytes. These findings indicate that miR-139 functions as a post-transcriptional regulator of MCPIP1 expression and enhances IL- 6 expression, which further upregulates miR-139 expression in OA chondrocytes. These results support our hypothesis that miR-139-mediated downregulation of MCPIP1 promotes IL- 6 expression in OA. Therefore, targeting miR-139 could be therapeutically beneficial in the management of $\mathrm{OA}$.

Experimental \& Molecular Medicine (2015) 47, e189; doi:10.1038/emm.2015.66; published online 9 October 2015

\section{INTRODUCTION}

Human osteoarthritis (OA) is a chronic, nonclassical inflammatory disease of diarthrodial synovial joints and a leading cause of disability in the adult population worldwide. Mechanical stress on the joints is a primary reason for the development of OA but genetic and environmental factors also have critical roles in its pathogenesis. ${ }^{1}$ Progressive loss of collagens and proteoglycans due to the concerted activity of matrix degrading metalloproteases MMP-1 (collagenase-1), MMP-3 (stromelysin-1), MMP-2 (72 kDa gelatinase), MMP-9 (92 kDa gelatinase), MMP13 (collagenase-3) and members of the aggrecanase family ADAMTS4 and 5 disturb the cartilage homeostasis and affect joint stability and function. Pro-inflammatory cytokines interleukin (IL)-1 $\beta$, tumor necrosis factor-alpha (TNF- $\alpha$ ) and IL-6, which are also found at high levels in the synovial fluid of OA patients, are believed to have important roles in the pathophysiology of OA by upregulating the expression of matrix-degrading MMPs and aggrecanases and downregulating the synthesis of type-II collagen and aggrecan, which are the building blocks of cartilage matrix. ${ }^{2,3}$

Cytokines mRNA turnover is extensively regulated by posttranscriptional mechanism. ${ }^{4}$ A recently identified protein MCPIP1 (ZC3H12A/RegnaseA, NM_025079) contains intrinsic RNAse activity that requires stem-loop structure located in the 3' UTR of the target mRNAs for degradation. ${ }^{5}$ Expression of MCPIP1 is induced by MCP-1 and inflammatory cytokines including TNF- $\alpha$ and IL- $1 \beta$ in monocytes and macrophages. ${ }^{6}$ We have recently demonstrated that IL- $1 \beta$ is also a potentinducer of MCPIP1 expression in human OA chondrocytes. ${ }^{7,8}$ Other studies have shown that MCPIP1 null mice develop severe autoimmune complexities resulting in death after few weeks of birth. ${ }^{9}$ Interestingly, macrophages from MCPIP1 null mice produce elevated levels of IL-6 (NM_000600). ${ }^{6,9,10}$ These data suggests that MCPIP1 may be an important posttranscriptional regulator of IL-6 expression in vivo.

Multiple species of small non-coding RNAs are involved in the regulation of gene expression. Among them micro-RNAs

Department of Anatomy and Neurobiology, Northeast Ohio Medical University, Rootstown, OH USA

Correspondence: Professor T Haqqi, Department of Anatomy and Neurobiology, Northeast Ohio Medical University, 4209, State Route 44, Room 144, Rootstown, OH 44272, USA. 
(miRNAs), which are small 19-22 long single stranded RNAs, bind with the 'seed sequence' located in the 3' UTR, 5' UTR or coding sequence of the target transcript and promote mRNA degradation or impede translation. ${ }^{11-13}$ It is widely believed that various biological processes including cellular differentiation, proliferation and development are regulated by miRNAs. ${ }^{12}$ Alteration in miRNAs expression has been reported in the pathophysiology of various diseases including OA. ${ }^{14,15}$ Cartilage-specific Dicer-null mice showed severe skeletal growth defects and premature death. ${ }^{16}$ More direct evidence of miRNAs role in OA pathogenesis is provided by miRNA-140-deficient mice, which develop age-related OA symptoms with loss of proteoglycan and fibrillation of articular cartilage. ${ }^{17}$ In addition, other miRNAs including miR-146, 9, 22, 27a/b, 602 and 608 have been demonstrated to have a significant role in regulating OA-related genes in chondrocytes in vitro. ${ }^{13-18}$

In a previous study, we demonstrated the miR-9-mediated regulation of MCPIP1 and IL-6 expression in human OA chondrocytes. ${ }^{7,8,19}$ Based on our data, we hypothesized that the 3' UTR of MCPIP1 mRNA might contain additional 'seed sequence' that may be targeted by other miRNAs and thus may be an important player in the post-transcriptional regulation of MCPIP1 expression in OA. Using TargetscanS algorithm, we identified a potential miR-139 'seed sequence' in the 3' UTR of MCPIP1 mRNA. Gene expression analysis using miRNA TaqMan assays in OA cartilage samples revealed that the expression of miR-139 was significantly higher in the damaged cartilage compared with smooth cartilage from the same OA patients $(P<0.05)$. Therefore, we investigated the role of miR-139 in regulating the post-transcriptional expression of MCPIP1 and the impact of such regulation on the expression of IL- 6 mRNA and protein in IL- $1 \beta$-stimulated OA chondrocytes. Overexpression of miR-139 downregulated the expression of MCPIP1 and increased the expression of IL-6 mRNA and protein and of MMP13 and ADAMTS4 mRNAs in OA chondrocytes. We also found that overexpression of miR-139 induces apoptosis in OA chondrocytes. Our data also demonstrated that the caspase $3 / 7$ activity was higher in OA chondrocytes with the overexpression of both miR-139 and miR-9. These findings indicate that the high levels of miR-9 and miR-139 expression detected in OA cartilage may contribute to the pathophysiology of OA by enhancing the expression of IL-6 and induction of apoptosis, and that targeting miR-139/9 may be beneficial in controlling disease progression.

\section{MATERIALS AND METHODS}

\section{Reagents}

Media and other reagents for chondrocyte culture were purchased from Life Technologies (Carlsbad, CA, USA). Pronase and collagenase were from Roche Diagnostics (Indianapolis, IN, USA). Recombinant human IL-1 $\beta$ and IL-6 were procured from R\&D Systems (Minneapolis, MN, USA). Antibodies specific for BCL-2 and $\beta$-Actin were purchased from Santa Cruz Biotechnology (Santa Cruz, CA, USA) and anti-BclX/l antibodies were obtained from Millipore (Billerica, MA, USA). Horseradish peroxidase-conjugated secondary antibodies were obtained from Pierce Biotechnology (Fisher Scientific, Waltham, MA, USA). Kits and other supplies for the preparation of total RNA were from Qiagen (Valencia, CA, USA). Caspase Glo 3/7 Assay kit was purchased from Promega (Madison, WI, USA). Human IL-6 PicoKine ELISA Kit was purchased from Booster Bio (Fremont, CA, USA).

\section{Cartilage samples and preparation of chondrocytes}

The study protocol to use de-identified, discarded human cartilage samples was reviewed and approved as 'non-human subject study under 45 CFR' by the Institutional Review Board of North East Ohio Medical University, Rootstown, OH and SUMMA Health Systems, Akron, OH. Knee cartilage of OA patients aged 45-70 years (eight OA cartilage samples, four males and four females, mean age $58 \pm 11.5$ years) who underwent total joint arthroplasty at Summa St Thomas Hospital, Akron, $\mathrm{OH}$ were used to prepare the chondrocytes by enzymatic digestion as previously described in several of our publications. ${ }^{13,20-22}$ Pieces of smooth cartilage were resected from the area with surface integrity without any irregularity (no staining with India ink, smooth cartilage), while damaged cartilage was obtained from area with gross erosions and stained positive with India ink. Some pieces of cartilage were stored in liquid nitrogen immediately for RNA isolation later and rest of the pieces were digested first with Pronase $\left(1 \mathrm{mg} \mathrm{ml}^{-1}\right)$ for $1 \mathrm{~h}$ and then with Collagenase $\left(1 \mathrm{mg} \mathrm{ml}^{-1}\right)$ and the isolated chondrocytes were plated in complete medium essentially as described previously. ${ }^{13,20,21,23}$

\section{Preparation of total RNA and gene expression analyses}

To prepare total RNA directly from cartilage samples, resected cartilage pieces were frozen in liquid nitrogen and grinded in a freezer mill in liquid nitrogen (Spex, Metuchen, NJ, USA) and the resulting powder was used to make total RNA using a commercially available kit (RNeasy Plus mini kit, Qiagen, Valencia, CA, USA). Total RNA from cultured chondrocytes was prepared by lysing the cells directly in the lysis buffer (RNeasy Plus mini kit) essentially according to the protocol provided with the kit. Single stranded complementary DNA was synthesized using the genomic DNA-free total RNA using the Quantitect Reverse Transcription kit (Qiagen) according to the manufacturer's protocol. Gene expression of COL2A1 (NM_001844), Aggrecan (NM_013227), COL10A1 (NM_008032), COL1A1 (NM_000088), MMP-13 (NM_002427), ADAMTS-4 (NM_005099), ADAMTS-5 (NM_007s038) and IL-6 (NM_000600) was quantified using TaqMan assays (Life Technologies). Expression of mature miRNAs was determined using miRNA TaqMan Assays. Expression levels were determined in one plate for each set of experiment. Values were normalized to the corresponding amounts of $\beta$-Actin or RNU6B expression level measured within the same plate. Relative expression levels were calculated using the $2^{-\Delta \Delta C T}$ method. ${ }^{24}$

\section{Chondrocytes treatment and transfections}

For each treatment 1.2 million primary OA chondrocytes were seeded into $3.5 \mathrm{~cm}$ dishes, and when $80 \%$ confluent chondrocytes were incubated in serum-free medium for $12 \mathrm{~h}$ and then treated with IL- $1 \beta$ for the indicated time point or with different concentrations of IL- 6 as indicated in the figure legend. OA chondrocytes were transfected with $200 \mathrm{~nm}$ control miRNA or miR-139 mimic or inhibitor using Amaxa Nucleofactor System and the chondrocyte Transfection kit according to the manufacturer's protocol (Lonza, Walkersville, MD, USA). Thirty-six hours post transfection, OA chondrocytes were stimulated with $2 \mathrm{ng} \mathrm{ml}^{-1}$ of IL- $1 \beta$ for an additional $24 \mathrm{~h}$. 


\section{Immunoblotting and caspase $3 / 7$ activity assay}

Primary OA chondrocytes were transfected as above using control miRNA or miR-9 or miR-139 mimetics. After treatment, OA chondrocytes were harvested and western blotting was performed essentially as describedbefore. ${ }^{25,26}$ Briefly OA chondrocytes were washed with cold PBS and lysed in ice-cold lysis buffer $(50 \mathrm{~mm}$ Tris $\mathrm{pH}$ 7.6, $400 \mathrm{~mm} \mathrm{NaCl}, 0.5 \% \mathrm{NP}-40,1 \mathrm{~mm}$ PMSF and $1 \times$ protease inhibitor cocktail (Roche). Lysates were clarified by centrifugation ( $15000 \mathrm{~g}$ for $15 \mathrm{~min}$ at $4{ }^{\circ} \mathrm{C}$ ) and the supernatant was transferred to another eppendorf tube. Equivalent amount of protein lysates $(25 \mu \mathrm{g})$ were resolved by $10 \%$ SDS-polyacrylamide gel electrophoresis and transferred to a polyvinylidene difluoride (PVDF) membrane (Hybond $\mathrm{P}$, Amersham Biosciences, Piscataway, NJ, USA) and the blots were incubated with anti-BCl2 (1:1000), anti-BClx/l (1:1000) or anti- $\beta$ Actin (1:5000) primary antibodies in TBS with Tween with 5\% bovine serum albumin overnight at $4{ }^{\circ} \mathrm{C}$. Blots were then incubated with the appropriate horseradish peroxidase-conjugated secondary antibodies and the antibody reactive proteins were visualized and analyzed using the Syngene Pxi imager and the Syngene Image analysis software (Syngene, Cambridge, UK), respectively. For miRNA-induced caspase activity, the assay was performed essentially as described by the manufacturer of the kit (Promega). Luminescence was measured in BioTek microplate reader (BioTek, Winooski, VT, USA).

\section{IL-6 ELISA assay}

IL-6 levels in culture supernatants from miR-139 mimic- or inhibitortransfected chondrocytes were determined using a human IL-6specific enzyme-linked immunosorbent assay (ELISA) kit according to manufacturer's instructions (Boster Immunoleader, Pleasonton, CA, USA). Values were derived using a standard curve prepared using recombinant human IL-6.

\section{Luciferase activity reporter assay}

The 3' UTR of MCPIP1 mRNA was amplified by PCR using primer pairs 5'-GTCAACTAGTCTCTCCTACAAGTCCCAGCA-3' and 5'-TGACAAGCTTTTGAAAGGGCTCACAAT GAT-3'. PCR was performed in a final volume of $50 \mu \mathrm{l}$ mixture containing $1 \times$ reaction buffer, $0.2 \mu \mathrm{M}$ of each primer, $200 \mu \mathrm{M}$ dNTPs, $1.5 \mathrm{mM} \mathrm{MgCl}_{2}$ and $2.5 \mathrm{U}$ of Platinum Taq DNA Polymerase (Life Technologies) and consisted of an initial denaturation step of $2 \mathrm{~min}$ at $94^{\circ} \mathrm{C}$, followed by 35 cycles of $94{ }^{\circ} \mathrm{C}$ for $30 \mathrm{~s}, 60^{\circ} \mathrm{C}$ for $30 \mathrm{~s}$ and $72{ }^{\circ} \mathrm{C}$ for $60 \mathrm{~s}$, with a final extension step of $5 \mathrm{~min}$ at $72{ }^{\circ} \mathrm{C}$. Amplified product was digested with HindIII and SpeI restriction endonucleases in the CutSmart buffer system (New England Biolabs, Ipswich, MA, USA) and cloned into the gel-purified pMIR-REPORT vector digested with the same enzymes. QuikChange II XL Site-Directed Mutagenesis Kit (Agilent Technologies, Santa Clara, CA, USA) was used to introduce the mutations into the miR-139 'seed sequence' located in the 3 ' UTR of the MCPIP-1 mRNA using the oligo 5'-CAAACCAAAGATAAACGTGGATTGG TTCTGG-3' (mutated nucleotides are in bold). Primary OA chondrocytes were nucleofected with the pMIR-REPORT vector containing either the wild-type or mutant 3' UTR of the MCPIP-1 mRNA as described above with miR-139 mimic or miR-control miRNAs. Thirty-six hours post transfection luciferase enzyme activity was measured using a commercially available kit (Promega). Renilla luciferase plasmid was co-transfected in each sample as transfection control and used to normalize the firefly luciferase activity values.

\section{Statistic analysis}

Each experiment was repeated three times using three independent patient samples. The values are presented as the mean \pm s.d. and the statistically significant difference between the experimental groups and controls was determined using Student's $t$-test. $P<0.05$ was considered to be statistically significant.

\section{RESULTS}

miR-139 was highly overexpressed in damaged OA cartilage and its expression was induced by IL-1 $\beta$ in primary $\mathrm{OA}$ chondrocytes

In our previous study, we have shown the reduced expression of MCPIP1 in damaged OA cartilage compared with that of the smooth cartilage. ${ }^{7,8}$ Close analyses of the MCPIP1 mRNA 3' UTR sequence revealed the existence of miRNA-139 'seed sequence' in the $3^{\prime}$ UTR of MCPIP1 transcript. This prompted us to investigate the dynamics of the miR-139 expression in damaged cartilage. MicroRNA-139 expression was significantly high in the damaged area of OA cartilage samples compared with the cartilage derived from the smooth cartilage from the same patients (Figure 1a). To mimic the pathological conditions, OA chondrocytes obtained from the smooth or damaged region of the OA cartilage were stimulated with IL-1 $\beta$ $\left(2 \mathrm{ng} \mathrm{ml}^{-1}\right)$ in a time-dependent manner and miR-139 expression was analyzed by TaqMan microRNA assay. Expression of miR-139 was induced significantly in OA chondrocytes from both the smooth or damaged cartilage post 12-h stimulation with IL-1 $\beta$ (Figures $1 \mathrm{~b}-\mathrm{c}$ ). These results indicate that miR-139 expression is IL-1 $\beta$ inducible and higher expression in damaged cartilage may be related to its exposure to inflammatory milieu that includes IL-1 $\beta$ in the diseased joint.

\section{Expression of marker genes in human OA chondrocytes cultured in monolayer}

To determine if monolayer culturing of chondrocytes results in alteration of chondrocyte marker genes, we determined the expression of COL2A1 (NM 001844), Aggrecan (NM_013227), COL10A1 (NM_008032) and COL1A1 (NM_000088) by TaqMan assays. As shown in Figure 1d, human chondrocytes cultured in monolayer for up to $72 \mathrm{~h}$ expressed COL2A1 and ACAN genes robustly with little or no expression of COL10A1 and COL1A1 detected at the time points analyzed. We thus conclude that in short-term (72 h) monolayer cultures, primary human OA chondrocytes maintain their phenotype.

Overexpression of $\mathrm{miR}-139$ alter the expression of MCPIP1 and IL-6 mRNA and protein in OA chondrocytes

Because of the presence of the miR-139 'seed sequence' in the 3'UTR of MCPIP1 mRNA, we determined whether miR-139 post-transcriptionally regulates the expression of MCPIP1 mRNA in OA chondrocytes. Overexpression of miR-139 inhibitor in $\mathrm{OA}$ chondrocytes significantly increased the expression of MCPIP1 mRNA (35\%) (Figure 2a; $P<0.05$ ). In contrast, overexpression of miR-139 decreased the levels of MCPIP1 transcripts (42\%) in the transfected OA chondrocytes 


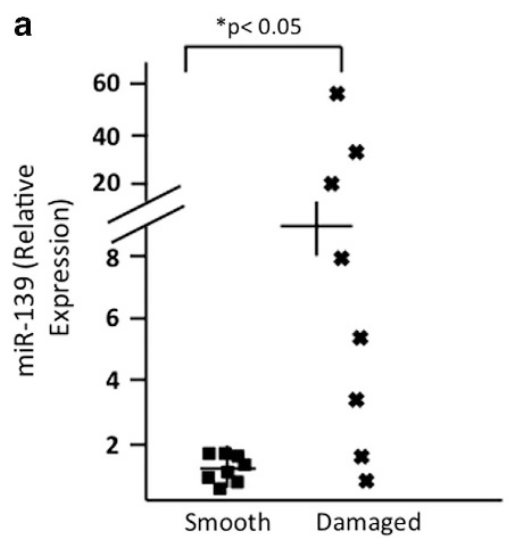

c

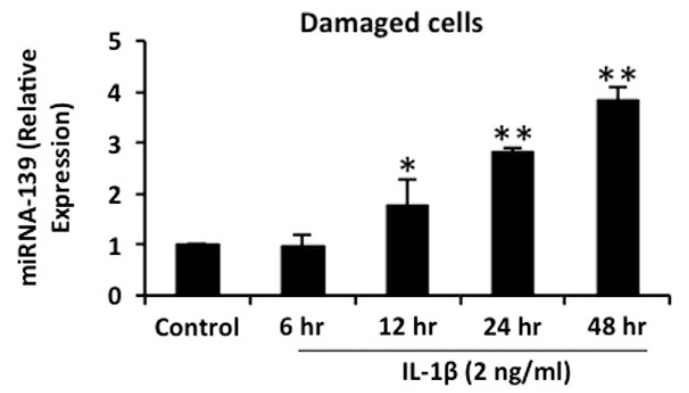

b

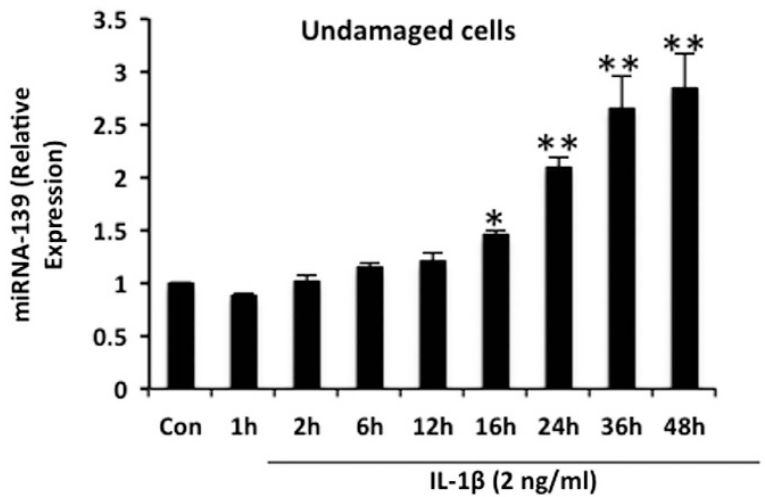

d

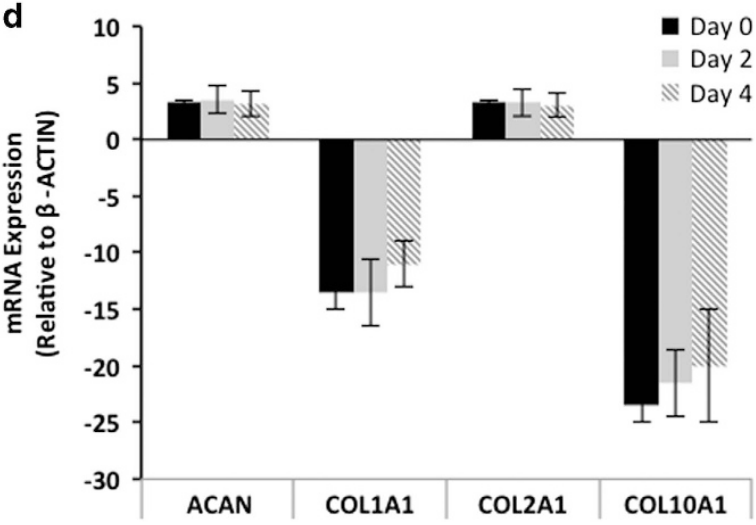

Figure 1 Expression of miR-139 is altered in damaged cartilage and is induced on IL-1 $\beta$ treatment. (a) Total RNA was isolated from smooth or damaged cartilage from OA patients, and miR-139 expression was measured by TaqMan assay. (b, c) Chondrocytes isolated

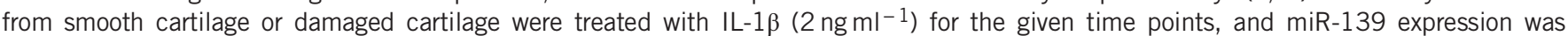
measured as above. RNU6B was used as an internal control. (d) Expression of marker genes in human OA chondrocytes cultured in monolayer was determined by TaqMan assay. $n=3$. ${ }^{*} P<0.05 ;{ }^{*} P<0.01$; paired Student's $t$-test.

(Figure 2a; $P<0.05)$. Since, MCPIP1 is a post-transcriptional regulator of IL-6 expression in OA chondrocytes as shown by us $^{7,8}$ and in other cell types by others, ${ }^{5,9}$ we determined the effect of miR-139 inhibition or overexpression on the expression of IL-6 mRNA and secreted protein. Inhibition of miR-139 decreased the expression of IL- 6 mRNA by $38 \%$ and secreted the IL- 6 protein by $40 \%$ in OA chondrocytes and culture supernatants, respectively (Figures $2 \mathrm{~b}, P<0.05$ ). However, overexpression of miR-139 increased the expression of IL- 6 mRNA by $36 \%$ and secreted IL- 6 protein by $56 \%$, which correlates with the downregulation of MCPIP1 mRNA expression and is in agreement with the above results (Figure 2c; $P<0.05)$. These data indicate that miR-139 is an important direct post-transcriptional regulator of MCPIP-1 and indirect post-transcriptional regulator of IL-6 expression in OA chondrocytes in vitro.

Overexpression of miR-139 alters the expression of catabolic genes but not the expression of anabolic genes in $\mathrm{OA}$ chondrocytes

Many well-characterized genes are known to be involved in the pathogenesis of OA. For example, catabolic proteins like metalloproteases and aggrecanases are overexpressed and degrade their respective targets in the cartilage matrix leading to cartilage breakdown in OA.,27 To assay the effect of miR-139 overexpression or inhibition in OA chondrocytes on the expression of anabolic and catabolic genes, we transfected OA chondrocytes with miR-139 mimics or inhibitors and stimulated with IL-1 $\beta$ as above and analyzed the mRNA expression of known anabolic and catabolic genes. Expression of anabolic genes ACAN, COL1A1 and COL10A1 was not altered but reduction was observed in the expression of COL2A1 as described in an earlier report ${ }^{28}$ (Figure 3a). However, ADAMTS4 and MMP13 showed increased mRNA expression in OA chondrocytes transfected with miR-139 mimics (32\% and 20\%, respectively) (Figure $3 \mathrm{~b} ; P<0.05$ ). Contrary to that, OA chondrocytes transfected with the miR-139 inhibitor showed repressed expression of MMP13 and ADAMTS4 mRNAs although no miR-139 seed sequence was identified in the $3^{\prime} \mathrm{UTR}$ of these mRNAs. This indicates that the inhibitory effect was not direct. Inhibition of the other assayed catabolic genes MMP3, MMP9, ADAMTS5 or TNF- $\alpha$ was not observed, which suggests that their expression is not regulated by miR-139 (Figure 3b). MMP13 and ADAMTS4 are 
a

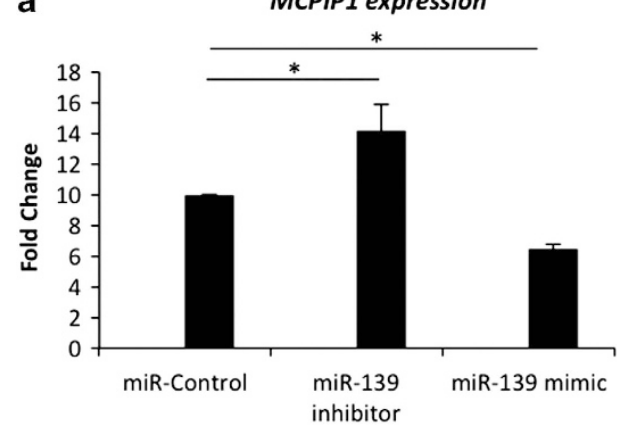

b

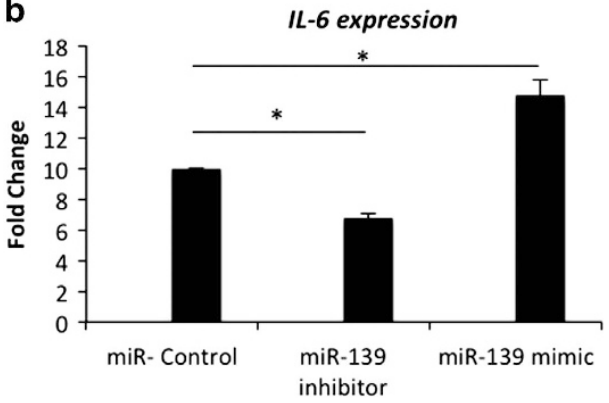

c

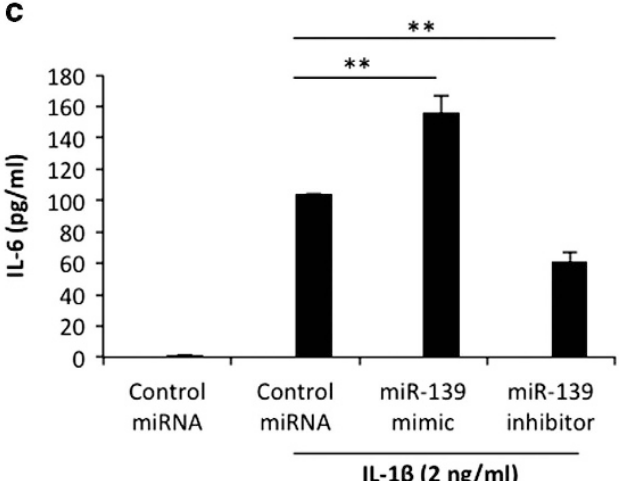

Figure 2 miR-139-mediated effect on the expression of MCPIP1 or IL-6 in human OA chondrocytes. OA chondrocytes were nucleofected with miR-139 mimic, miR-139 inhibitor and miR-control. Thirty-six hours later, chondrocytes were stimulated with $2 \mathrm{ng} \mathrm{ml}-1$ IL-1 $\beta$ for $24 \mathrm{~h}$. RNA was isolated from the chondrocytes, and complementary DNA was generated. mRNA expression was measured by TaqMan assays specific for MCPIP1 (a) and IL-6 (b) mRNAs. Gene-specific mRNA expression was normalized against $\beta$-actin expression. (c) Sandwich ELISA was performed to determine the level of IL-6 in culture supernatant. $n=3$. ${ }^{*} P<0.05$; ${ }^{* *} P<0.01$; paired Student's t-test.

known to be induced by IL-6, ${ }^{29-31}$ therefore the changes observed in their expression may be due to the increased or decreased levels of IL-6 in OA chondrocytes with overexpression of miR-139 mimics or miR-139 inhibitor, respectively.

miR-139 target the 'seed sequence' in the 3 ' UTR of MCPIP1 mRNA

To confirm that miR-139 interacts with the 'seed sequence' located in the $3^{\prime}$ UTR of the MCPIP-1 mRNA, we performed the luciferase enzyme activity reporter assay containing either the wild-type or mutated miR-139 'seed sequence'. For these studies, wild-type or mutant MCPIP1-3'UTR reporter construct was co-transfected into OA chondrocytes with miR-139 mimic or miR-control and the luciferase enzyme activity was measured and normalized against Renilla luciferase. As shown, the luciferase enzyme activity was significantly reduced in $\mathrm{OA}$ chondrocytes with the overexpression of miR-139 (Figure 4). However, inhibition of the luciferase enzyme activity was abolished when reporter vector containing the mutated site in the 3' UTR was co-transfected with miR-139 mimics (Figure 4). This data confirms that miR-139 interacts with the 'seed sequence' located in the 3' UTR of the MCPIP1 mRNA.

\section{IL-6 increased the expression of miR-139}

As shown previously IL-1 $1 \beta$-induced IL-6 expression is controlled by MCPIP1, which in turn is regulated by miR- $9^{7}$ and miR-139 (this report). Previously, we have shown the IL-6 induced the expression of miR-9. ${ }^{7,8}$ To determine whether IL-6 also induces the expression of miR-139, we treated the OA chondrocytes cultures with increasing concentrations of IL-6. Twenty-four hours post induction, expression of miR-139 was measured by TaqMan miRNA assay. Expression of miR-139 was significantly induced at $20 \mathrm{ng} \mathrm{ml}^{-1}$ concentration that was further enhanced in a dose-dependent manner (Figure 5). This suggests that IL- 6 also has a role in increasing the miR-139 expression in OA chondrocytes, which probably is important in the feedback loop inhibition of MCPIP1 expression to maintain high levels of IL-6 expression in OA chondrocytes.

miR-139 and miR-9 induce apoptosis in OA chondrocytes To further investigate the biological functions of miR-139 and miR-9 in OA chondrocytes, we overexpressed these two microRNAs individually or in combination in OA chondrocytes. Expression of anti-apoptotic protein BCL2 (NM_000633) or BCLXL (NM_001191) in OA chondrocytes with the overexpression of miR-9 and miR-139 was decreased (Figure 6a) although we could not identify 'seed sequence' for either miR-139 or miR-9 in the $3^{\prime}$ UTR of these mRNAs. This suggests that the inhibitory effect may be indirect but contributory to the chondrocyte apoptosis in OA. This is supported by our data demonstrating that caspase $3 / 7$ activity in miR-9 or miR-139 mimic-transfected chondrocytes was significantly high compared with the activity level detected in OA chondrocytes transfected with control miRNA. However 

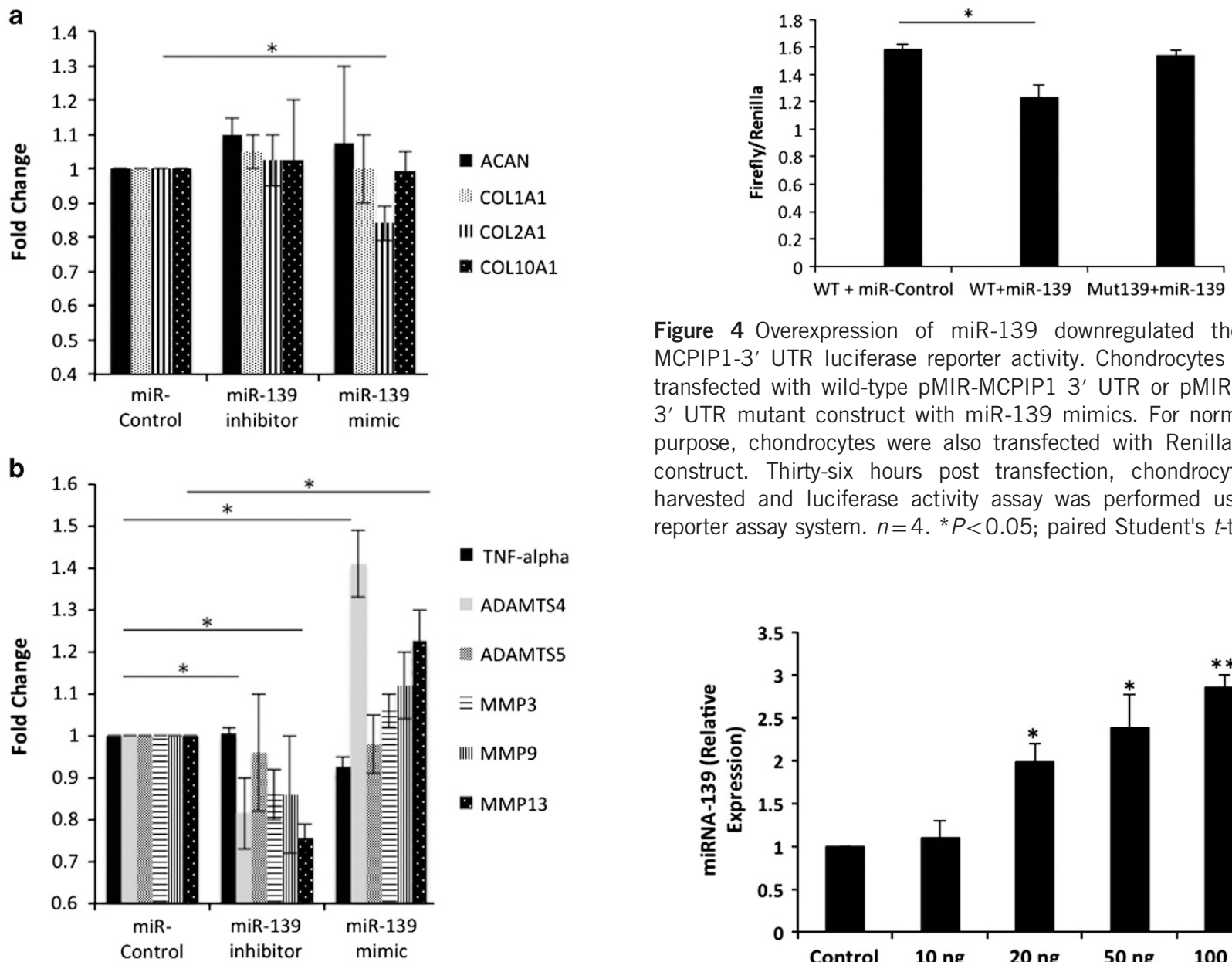

Figure 3 Effect of miR-139 on the expression of human OA marker genes. OA chondrocytes were nucleofected with miR-139 mimic, miR-139 inhibitor or miR-control. Thirty-six hours later, chondrocytes were stimulated with $2 \mathrm{ng} \mathrm{ml}^{-1} \mathrm{IL}-1 \beta$ for $24 \mathrm{~h}$. RNA was isolated from the chondrocytes, and complementary DNA was generated. mRNA expression of anabolic (a) or catabolic (b) markers were quantified by TaqMan assays. $\beta$-Actin was used as an internal control. $n=3$. ${ }^{*} P<0.05$; paired Student's $t$-test.

this effect was more prominent when both the miRNAs were co-transfected (Figure 6b).

\section{DISCUSSION}

In the present study for the first time, we have shown the overexpression of miR-139 in the damaged OA cartilage compared with smooth OA cartilage from the same patients and the IL-1 $\beta$-induced expression of miR-139 in OA chondrocytes. Upregulation of miR-139 in damaged OA cartilage compared with smooth cartilage suggests that it has a pathophysiological role in the OA. Interestingly, overexpression of miR-139 decreased the expression of MCPIP1 and hence the observed increase in the IL-6 expression. This is in agreement with our previous findings where we showed that MCPIP1 is a negative regulator of IL-6 expression in OA chondrocytes. Furthermore, we found that the overexpression of miR-139

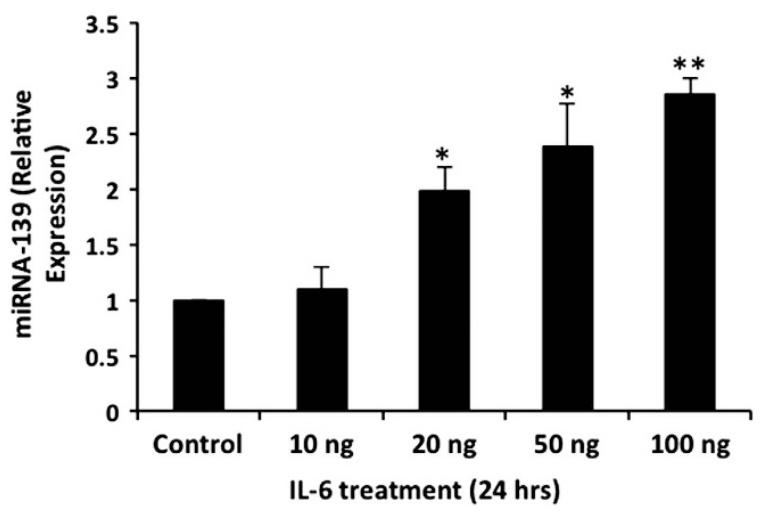

Figure 5 Expression of miR-139 was induced on IL- 6 treatment. $\mathrm{OA}$ chondrocytes were treated with increasing doses of IL-6 for $24 \mathrm{~h}$. miR-139 expression was measured by TaqMan assay. RNU6B was used as an internal control. $n=3$. ${ }^{*} P<0.05$; ${ }^{* *} P<0.01$; paired Student's $t$-test.

induced the expression of catabolic markers of OA pathogenesis but the expression of anabolic genes, except that of COL2A1, in OA chondrocytes was not changed. This may be related to the increased expression of IL-6, which is a known inducer of catabolic genes in OA. Of note, we also demonstrated that in OA chondrocytes with overexpression of both miR-139 and miR-9, expression of BCL2 and BCLXL was downregulated and that these chondrocytes also had enhanced caspase 3/7 activity levels. These data are suggestive of a role of miR-139 and miR-9 in the induction of apoptosis in OA chondrocytes. Since no conserved binding site for either miR-139 or miR-9 in the 3' UTR of BCL2 or BCLXL mRNAs was found, the observed effect appears to be indirect but this needs to be investigated further. In summary, our data strongly suggest that overexpressed miR-139 induces catabolic gene expression and in association with miR-9 induces apoptosis of chondrocytes, which could contribute in the pathogenesis of OA. 
a
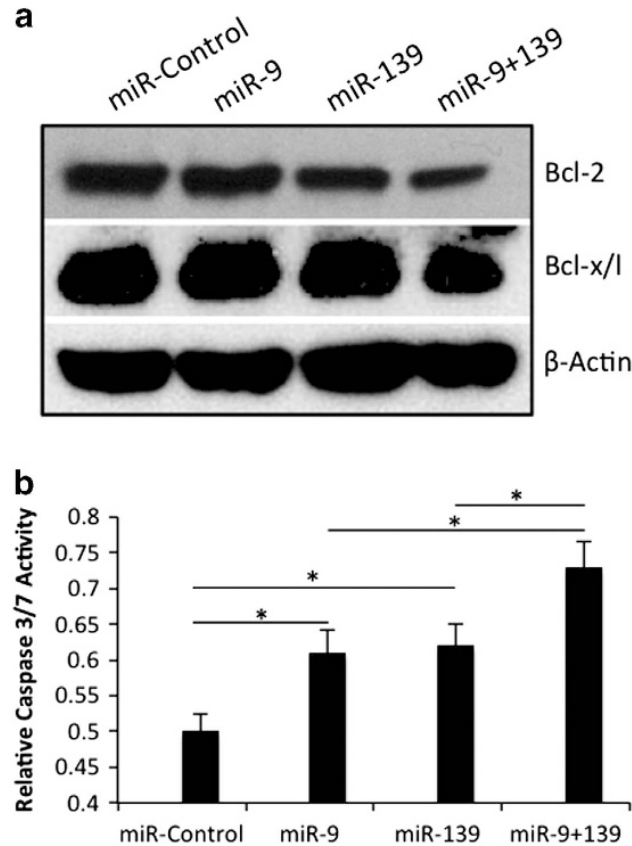

Figure 6 Overexpression of miR-139 increased the caspase 3/7 activity in $\mathrm{OA}$ chondrocytes, which was enhanced in the presence of miR-9. (a) Human OA chondrocytes were transfected with control miR, miR-9, miR-139 alone or in combination. Twenty-four hours later, $\mathrm{OA}$ chondrocytes were induced with $2 \mathrm{ng} \mathrm{ml}^{-1} \mathrm{IL}-1 \beta$ for an additional $36 \mathrm{~h}$. Lysates were prepared and protein was separated by SDS-polyacrylamide gel electrophoresis. Western blotting was performed using specific antibodies. (b) Chondrocytes were transfected as above. Lysates were prepared and caspase 3/7 activity was assayed using Caspase Glo 3/7 Assay kit according to the instructions provided by the manufacturer. $n=4$. ${ }^{*} P<0.05$; paired Student's $t$-test.

Cytokines such as TNF- $\alpha$ IL- $1 \beta$ GM-CSF, IL-2 and IL-17 are known to be regulated post-transcriptionally in macrophages or in T-lymphocytes by AU-rich element-binding proteins including TTP, HuR and AUF1. ${ }^{32}$ TTP binds to AU-rich elements present in the IL- 6 mRNA and recruits deadenylases to the complex, which activates the mRNA decay process. ${ }^{33}$ On the other hand AUF1 requires putative RNA stem loop structure and AU-rich element for IL-6 mRNA destabilization. ${ }^{34}$ Studies performed with MCPIP1 in macrophages and T lymphocytes also showed that MCPIP1 degrades IL-6 mRNA through a similar mode of action. ${ }^{35,36}$ In the present study, we identify another layer of regulation of IL-6 expression by demonstrating that miR-139 mediated decay of MCPIP1 mRNA is an important event in the enhanced IL-6 expression in IL-1 $\beta$-stimulated human OA chondrocytes. Expression of both miR-9 and miR-139 was upregulated in damaged OA cartilage and treatment of smooth- or damaged cartilage-derived chondrocytes by IL- $1 \beta$ significantly enhanced the levels of miR-139 expression in a time-dependent manner. ${ }^{7,8}$ Also miR-9 and miR-139 both have been implicated in cell proliferation and apoptosis in multiple cell types. Increasing miR-9 expression levels in breast cancer cells induced anti-proliferative and pro-apoptotic activity. ${ }^{37}$ miR-9 inhibits the proliferation of glioma cells by targeting cyclic AMP response element-binding protein (CREB), and in another study the authors found that miR-9 inhibits proliferation during neurogenesis via its action on $3^{\prime}$ UTRs of her6 and zic5 mRNAs. ${ }^{38,39}$ Similarly upregulation of miR-139 also suppressed the proliferation and enhanced temozolomideinduced apoptosis in glioma. ${ }^{40}$ In addition, the ectopic expression of miR-139 in human CRC cells decreased cell growth, whereas the silencing of miR-139 promoted cell growth. ${ }^{41} \mathrm{We}$ for the first time here show the pro-apoptotic activity of miR-9 and miR-139 in OA chondrocytes, suggesting high level of these two microRNAs may promote apoptosis in OA chondrocytes. However, it is fair to say that miR-9 and miR-139 could have been targeting multiple mRNAs, therefore the proapoptotic effect could have been due to interference with rather a complex interaction of multiple pathways.

Although the etiology of OA induction is not established but several studies have demonstrated the presence of inflammatory cytokines IL- $1 \beta$, IL- 6 and TNF- $\alpha$ in the OA joint cartilage, which exert a catabolic effect and thus are important contributors to the disease pathogenesis. IL-6 is a proinflammatory cytokine, which activates the transcription of its target genes via formation of an IL-6 receptor (IL-6R) complex involving a membrane-bound IL-6R, soluble IL-6R and gp130 followed by activation of STAT1/STAT3 pathway. ${ }^{42}$ IL-6 has been shown to upregulate the expression of catabolic enzymes MMP-1 and MMP-13 in human and bovine cartilage explants. ${ }^{43,44}$ Contrary to that IL-6 inhibits anabolic process by suppressing expression of type II collagen. ${ }^{28}$ Multiple studies showed the levels of IL-6 are elevated in the synovial fluid and serum of RA or in OA patients when compared with normal healthy controls. ${ }^{45,46}$ In Hartley guinea pig model of OA, IL-6 levels in serum were positively correlated with histological score of $\mathrm{OA}$, which was independent of age or weight. ${ }^{47}$ Another report by Tonchera et al. ${ }^{48}$ concluded that serum levels of IL-6 were significantly higher in patients with active OA compared with healthy subjects. Furthermore, there is a direct correlation in IL-6 expression level and clinical outcome of rheumatoid arthritis, and blocking of IL- 6 function has emerged a new therapeutic strategy for RA and related inflammatory disease. ${ }^{49}$ The correlation between the expression of miR-139, MCPIP1 and IL-6 was established by overexpressing specific mimics and antagomiRs. These results demonstrated that miR-139 overexpression suppressed the expression of MCPIP1 mRNA and protein, and increased the expression of IL- 6 mRNA and protein. Importantly, we also observed that the expression of some OA catabolic marker genes was also affected specifically of MMP13, which could be related to the changes in the expression of IL-6 in OA chondrocytes.

In summary, our study provides strong evidence of miR-139-mediated direct post-transcriptional regulation of MCPIP1 and indirect post-transcriptional regulation of IL-6 expression in OA chondrocytes. Increased expression of catabolic markers of OA and enhanced activity levels of caspases 3/7 were also promoted when miR-139 and miR-9 were overexpressed in OA chondrocytes, suggesting a role of these 
microRNAs in the pathogenesis of OA. Therefore, targeting miR-9 and 139 may provide benefit in the management of OA pathogenesis.

\section{CONFLICT OF INTEREST}

The authors declare no conflict of interest.

\section{ACKNOWLEDGEMENTS}

This work was supported in part by National Institute of Health grants (RO1-AT-005520; RO1-AT-007373; RO1-AR067056) and funds from the Northeast Ohio Medical University to TMH.

1 Goldring MB, Marcu KB. Epigenomic and microRNA-mediated regulation in cartilage development, homeostasis, and osteoarthritis. Trends Mol Med 2012; 18: 109-118.

2 Goldring SR, Goldring MB. The role of cytokines in cartilage matrix degeneration in osteoarthritis. Clin Orthop Relat Res 2004; 427: S27-S36.

3 Haseeb A, Haqqi TM. Immunopathogenesis of osteoarthritis. Clin Immunol 2013; 146: 185-196.

4 White EJ, Brewer G, Wilson GM. Post-transcriptional control of gene expression by AUF1: mechanisms, physiological targets, and regulation. Biochim Biophys Acta 2013; 1829: 680-688.

5 Iwasaki H, Takeuchi O, Teraguchi S, Matsushita K, Uehata T, Kuniyoshi K et al. The IkappaB kinase complex regulates the stability of cytokineencoding mRNA induced by TLR-IL-1R by controlling degradation of regnase-1. Nat Immunol 2011; 12: 1167-1175.

6 Mizgalska D, Wegrzyn P, Murzyn K, Kasza A, Koj A, Jura J et al. Interleukin1 -inducible MCPIP protein has structural and functional properties of RNase and participates in degradation of IL-1beta mRNA. FEBS J 2009; 276: 7386-7399.

7 Makki MS, Haseeb A, Haqqi TM. Micro RNA-9 (miR-9) promote IL-6 expression by targeting MCPIP1 in IL-1 $\beta$-stimulated human chondrocytes. Ind J Rheumatol 2014; 9: S4.

8 Makki MS, Haseeb A, Haqqi TM. MCPIP1 regulates the expression of interleukin-6 in human osteoarthritis chondrocytes. Osteoarthr Cartil 2014; 22: S166

9 Matsushita K, Takeuchi O, Standley DM, Kumagai Y, Kawagoe T, Miyake T et al. Zc3h12a is an RNase essential for controlling immune responses by regulating mRNA decay. Nature 2009; 458: 1185-1190.

10 Zhou L, Azfer A, Niu J, Graham S, Choudhury M, Adamski FM et al. Monocyte chemoattractant protein-1 induces a novel transcription factor that causes cardiac myocyte apoptosis and ventricular dysfunction. Circ Res 2006; 98: 1177-1185.

11 Farh KK, Grimson A, Jan C, Lewis BP, Johnston WK, Lim LP et al. The widespread impact of mammalian MicroRNAs on mRNA repression and evolution. Science 2005; 310: 1817-1821.

12 Bartel DP. MicroRNAs: genomics, biogenesis, mechanism, and function. Cell 2004; 116: 281-297.

13 Akhtar N, Rasheed Z, Ramamurthy S, Anbazhagan AN, Voss FR, Haqqi TM. MicroRNA-27b regulates the expression of matrix metalloproteinase 13 in human osteoarthritis chondrocytes. Arthritis Rheum 2010; 62 1361-1371.

14 Swingler TE, Wheeler G, Carmont V, Elliott HR, Barter MJ, Abu-Elmagd M et al. The expression and function of microRNAs in chondrogenesis and osteoarthritis. Arthritis Rheum 2012; 64: 1909-1919.

15 Jones SW, Watkins G, Le Good N, Roberts S, Murphy CL, Brockbank SM et al. The identification of differentially expressed microRNA in osteoarthritic tissue that modulate the production of TNF-alpha and MMP13. Osteoarthr Cartil 2009; 17: 464-472.

16 Kobayashi T, Lu J, Cobb BS, Rodda SJ, McMahon AP, Schipani E et al. Dicer-dependent pathways regulate chondrocyte proliferation and differentiation. Proc Natl Acad Sci USA 2008; 105: 1949-1954.

17 Miyaki S, Sato T, Inoue A, Otsuki S, Ito $\mathrm{Y}$, Yokoyama $\mathrm{S}$ et al. MicroRNA-140 plays dual roles in both cartilage development and homeostasis. Genes Dev 2010; 24: 1173-1185.

18 Miyaki S, Asahara H. Macro view of microRNA function in osteoarthritis. Nat Rev Rheumatol 2012; 8: 543-552.
19 Akhtar N, Makki MS, TM Haqqi. MicroRNA-602 and MicroRNA-608 regulate sonic hedgehog expression via target sites in the coding region in human chondrocytes. Arthritis Rheumatol 2015; 67: 423-434.

20 Makki MS, Haseeb A, Haqqi TM. MicroRNA-9 promotes IL- 6 expression by inhibiting MCPIP1 expression in IL-1 $\beta$-stimulated human chondrocytes. Arthritis Rheumatol (e-pub ahead of print 27 April 2015; doi:10.1002/ art.39173).

21 Haseeb A, Makki MS, Haqqi TM. Modulation of ten-eleven translocation 1 (TET1), Isocitrate Dehydrogenase (IDH) expression, alpha-Ketoglutarate (alpha-KG), and DNA hydroxymethylation levels by interleukin-1beta in primary human chondrocytes. J Biol Chem 2014; 289: 6877-6885.

22 Rasheed Z, Akhtar N, Haqqi TM. Pomegranate extract inhibits the interleukin-1beta-induced activation of MKK-3, p38alpha-MAPK and transcription factor RUNX-2 in human osteoarthritis chondrocytes. Arthritis Res Ther 2010; 12: R195.

23 Rasheed Z, Anbazhagan AN, Akhtar N, Ramamurthy S, Voss FR, Haqqi TM. Green tea polyphenol epigallocatechin-3-gallate inhibits advanced glycation end product-induced expression of tumor necrosis factor-alpha and matrix metalloproteinase-13 in human chondrocytes. Arthritis Res Ther 2009; 11: R71.

24 Livak KJ, Schmittgen TD. Analysis of relative gene expression data using real-time quantitative PCR and the 2(-Delta Delta C(T)) Method. Methods 2001; 25: 402-408.

25 Makki MS, Heinzel T, Englert C. TSA downregulates Wilms tumor gene 1 (Wt1) expression at multiple levels. Nucleic Acids Res 2008; 36: 4067-4078.

26 Shahidul Makki M, Cristy Ruteshouser E, Huff V. Ubiquitin specific protease 18 (Usp18) is a WT1 transcriptional target. Exp Cell Res 2013; 319: 612-622

27 Rousseau J, Garnero P. Biological markers in osteoarthritis. Bone 2012; 51: 265-277.

28 Poree B, Kypriotou M, Chadjichristos C, Beauchef G, Renard E, Legendre F et al. Interleukin-6 (IL-6) and/or soluble IL-6 receptor downregulation of human type II collagen gene expression in articular chondrocytes requires a decrease of Sp1.Sp3 ratio and of the binding activity of both factors to the COL2A1 promoter. J Biol Chem 2008; 283: 4850-4865.

29 Mimata Y, Kamataki A, Oikawa S, Murakami K, Uzuki M, Shimamura T et al. Interleukin- 6 upregulates expression of ADAMTS-4 in fibroblast-like synoviocytes from patients with rheumatoid arthritis. Int $J$ Rheum Dis 2012; 15: 36-44.

30 Wahlgren J, Maisi P, Sorsa T, Sutinen M, Tervahartiala T, Pirila E et al. Expression and induction of collagenases (MMP-8 and -13) in plasma cells associated with bone-destructive lesions. J Pathol 2001; 194 217-224.

31 Hashizume M, Mihara M. Desirable effect of combination therapy with high molecular weight hyaluronate and NSAIDs on MMP production. Osteoarthr Cartil 2009; 17: 1513-1518.

32 Anderson P. Post-transcriptional control of cytokine production. Nat Immunol 2008; 9: 353-359.

33 Zhao W, Liu M, D'Silva NJ, Kirkwood KL. Tristetraprolin regulates interleukin-6 expression through p38 MAPK-dependent affinity changes with mRNA 3' untranslated region. J Interferon Cytokine Res 2011; 31: 629-637.

34 Paschoud S, Dogar AM, Kuntz C, Grisoni-Neupert B, Richman L, Kuhn LC. Destabilization of interleukin- 6 mRNA requires a putative RNA stem-loop structure, an AU-rich element, and the RNA-binding protein AUF1. Mol Cell Biol 2006; 26: 8228-8241.

35 Li M, Cao W, Liu H, Zhang W, Liu X, Cai Z et al. MCPIP1 downregulates IL-2 expression through an ARE-independent pathway. PloS One 2012; 7: e49841.

36 Uehata T, Akira S. mRNA degradation by the endoribonuclease Regnase-1/ ZC3H12a/MCPIP-1. Biochim Biophys Acta 2013; 1829: 708-713.

37 Selcuklu SD, Donoghue MT, Rehmet K, de Souza Gomes M, Fort A Kovvuru P et al. MicroRNA-9 inhibition of cell proliferation and identification of novel miR-9 targets by transcriptome profiling in breast cancer cells. J Biol Chem 2012; 287: 29516-29528.

38 Tan X, Wang S, Yang B, Zhu L, Yin B, Chao T et al. The CREB-miR-9 negative feedback minicircuitry coordinates the migration and proliferation of glioma cells. PloS One 2012; 7: e49570.

39 Coolen M, Thieffry D, Drivenes O, Becker TS, Bally-Cuif L. miR-9 controls the timing of neurogenesis through the direct inhibition of antagonistic factors. Dev Cell 2012; 22: 1052-1064.

40 Li RY, Chen LC, Zhang HY, Du WZ, Feng Y, Wang HB et al. MiR-139 inhibits Mcl-1 expression and potentiates TMZ-induced apoptosis in glioma. CNS Neurosci Ther 2013; 19: 477-483. 
41 Guo H, Hu X, Ge S, Qian G, Zhang J. Regulation of RAP1B by miR-139 suppresses human colorectal carcinoma cell proliferation. Int J Biochem Cell Biol 2012; 44: 1465-1472.

42 Zhong Z, Wen Z, Darnell JE Jr. Stat3: a STAT family member activated by tyrosine phosphorylation in response to epidermal growth factor and interleukin-6. Science 1994; 264: 95-98.

43 Cawston TE, Curry VA, Summers CA, Clark IM, Riley GP, Life PF et al. The role of oncostatin $\mathrm{M}$ in animal and human connective tissue collagen turnover and its localization within the rheumatoid joint. Arthritis Rheum 1998; 41: 1760-1771.

44 Rowan AD, Koshy PJ, Shingleton WD, Degnan BA, Heath JK, Vernallis AB et al. Synergistic effects of glycoprotein 130 binding cytokines in combination with interleukin-1 on cartilage collagen breakdown. Arthritis Rheum 2001; 44: 1620-1632.

45 Kaneko S, Satoh T, Chiba J, Ju C, Inoue K, Kagawa J. Interleukin-6 and interleukin-8 levels in serum and synovial fluid of patients with osteoarthritis. Cytokines Cell Mol Ther 2000; 6: 71-79.

46 Houssiau FA, Devogelaer JP, Van Damme J, de Deuxchaisnes CN, Van Snick J. Interleukin-6 in synovial fluid and serum of patients with rheumatoid arthritis and other inflammatory arthritides. Arthritis Rheum 1988; 31: 784-788.
47 Huebner JL, Seifer DR, Kraus VB. A longitudinal analysis of serum cytokines in the Hartley guinea pig model of osteoarthritis. Osteoarthr Cartil 2007; 15: 354-356.

48 Toncheva A, Remichkova M, Ikonomova K, Dimitrova P, Ivanovska N. Inflammatory response in patients with active and inactive osteoarthritis. Rheumatol Int 2009; 29: 1197-1203.

49 Ding C, Jones G. Anti-interleukin-6 receptor antibody treatment in inflammatory autoimmune diseases. Rev Recent Clin Trials 2006; 1: 193-200.

(c) (1) (8) This work is licensed under a Creative Commons Attribution-NonCommercial-NoDerivs 4.0 International License. The images or other third party material in this article are included in the article's Creative Commons license, unless indicated otherwise in the credit line; if the material is not included under the Creative Commons license, users will need to obtain permission from the license holder to reproduce the material. To view a copy of this license, visit http://creativecommons.org/licenses/by-nc-nd/4.0/ 